\title{
Improving Fourier Partial Sum Approximation for Discontinuous Functions Using a Weight Function
}

\author{
Beong In Yun \\ Department of Mathematics, Kunsan National University, Gunsan, Republic of Korea \\ Correspondence should be addressed to Beong In Yun; paulllyun@gmail.com
}

Received 1 September 2017; Revised 16 October 2017; Accepted 19 October 2017; Published 22 November 2017

Academic Editor: Roberto Barrio

Copyright (C) 2017 Beong In Yun. This is an open access article distributed under the Creative Commons Attribution License, which permits unrestricted use, distribution, and reproduction in any medium, provided the original work is properly cited.

\begin{abstract}
We introduce a generalized sigmoidal transformation $w_{m}(r ; x)$ on a given interval $[a, b]$ with a threshold at $x=r \in(a, b)$. Using $w_{m}(r ; x)$, we develop a weighted averaging method in order to improve Fourier partial sum approximation for a function having a jump-discontinuity. The method is based on the decomposition of the target function into the left-hand and the right-hand part extensions. The resultant approximate function is composed of the Fourier partial sums of each part extension. The pointwise convergence of the presented method and its availability for resolving Gibbs phenomenon are proved. The efficiency of the method is shown by some numerical examples.
\end{abstract}

\section{Introduction}

For a function $f$ having a jump-discontinuity, every traditional spectral partial sum approximation will not converge uniformly on any interval containing the discontinuity. This deficiency of the spectral approximation results in the socalled Gibbs phenomenon which shows nonvanishing spikes near the discontinuity $[1,2]$. There are lots of methods to overcome the problem such as the Fourier-Gegenbauer method [3-5], the inverse reconstruction $[6,7]$, and the adaptive filtering method [8-11]. But most existing methods need a large number of terms to support high accuracy.

In this work, focusing on the Fourier partial sum approximation for a piecewise smooth function $f$ having a jumpdiscontinuity $\xi$, we aim to develop a constructive approximation procedure which is available for eliminating the Gibbs phenomenon near the discontinuity. First, in the following section, we introduce the so-called generalized sigmoidal transformation $w_{m}(r ; x)$ with a threshold $r=\xi$. Using $w_{m}$, we decompose the target function $f$ into the left-hand part extension $\tilde{f}_{L}$ and the right-hand part extension $\tilde{f}_{R}$ as described in Section 3. Then we combine Fourier partial sums of $\widetilde{f}_{L}$ and $\widetilde{f}_{R}$ by the form of a weighted average, $F_{n}$, as given in (26) in Section 4. We prove the pointwise convergence of the presented approximation $F_{n}$ to the discontinuous function $f$ over the whole interval. Moreover, it is shown that the asymptotic version of $F_{n}$ which is composed of uniform convergent partial sums will overcome the Gibbs phenomenon. This means that $F_{n}$ can sufficiently resolve the problem of inevitable wiggles of the traditional Fourier partial sum approximation near the jump-discontinuity. In addition, numerical results for some examples show the availability of the presented method.

\section{A Generalized Sigmoidal Transformation}

For a given interval $[a, b]$ and some interior point $a<r<b$, referring to the literature [12], we introduce the real valued function

$$
\begin{array}{r}
w_{m}(r ; x)=\frac{(x-a)^{m}}{(x-a)^{m}+\{((r-a) /(b-r))(b-x)\}^{m}}, \\
a \leq x \leq b
\end{array}
$$

for an integer $m \geq 1$. It was used for cumulative averaging method for piecewise polynomial interpolations in [12]. We call $w_{m}(x)=w_{m}(r ; x)$ a generalized sigmoidal transformation of order $m$ with a threshold $r$.

We can observe the basic properties of $w_{m}$ as follows:

(i) The special case of $r=(a+b) / 2$, with $a=0$ and $b=1$, is

$$
w_{m}(x)=\frac{x^{m}}{x^{m}+(1-x)^{m}},
$$




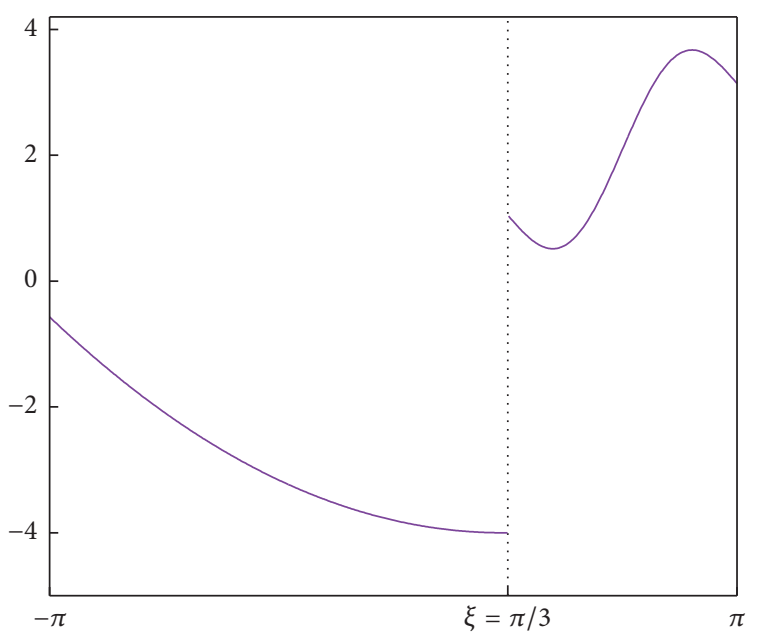

$(x)$

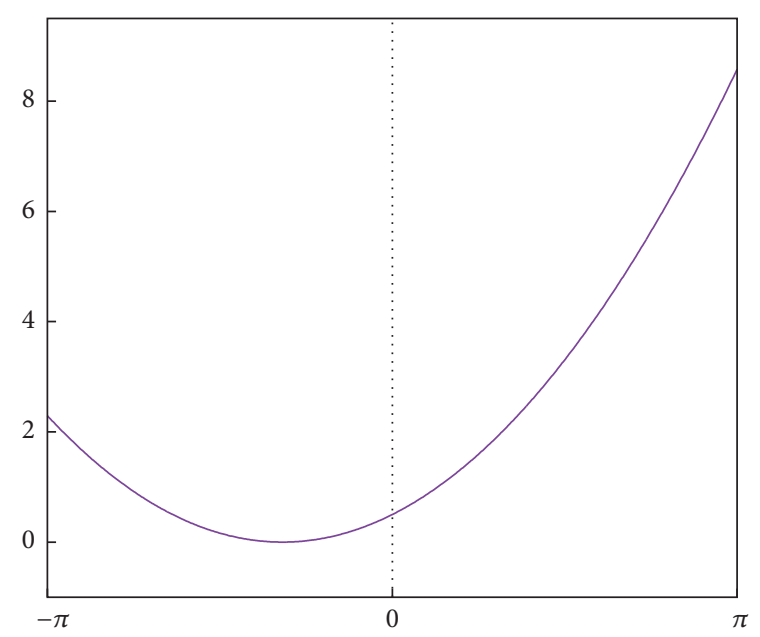

$(x)$

(b) $f_{2}(x)$

FIGURE 1: Graphs of test functions.

which is the same with the elementary sigmoidal transformation proposed in $[13,14]$.

(ii) Values of $w_{m}(x)$ at the points $x=a, r$, and $b$ are

$$
\begin{aligned}
& w_{m}(a)=0, \\
& w_{m}(r)=\frac{1}{2}, \\
& w_{m}(b)=1
\end{aligned}
$$

independently of the parameters $a, b, r$, and $m$. In addition, $w_{m}(x)$ is strictly increasing on the interval $[a, b]$ because the derivative of $w_{m}(x)$ with respect to $x$ satisfies

$$
w_{m}^{\prime}(x)
$$

$$
\begin{aligned}
& =\frac{m(b-a)(b-r)^{m}(r-a)^{m}(b-x)^{m-1}(x-a)^{m-1}}{\left\{(r-a)^{m}(b-x)^{m}+(b-r)^{m}(x-a)^{m}\right\}^{2}} \\
& >0
\end{aligned}
$$

for all $a<x<b$.

(iii) Asymptotic behavior of $w_{m}(x)$ near the end points $a$ and $b$ is

$$
w_{m}(x)= \begin{cases}O\left(\left[\frac{x-a}{r-a}\right]^{m}\right), & x<r \\ 1+O\left(\left[\frac{b-x}{b-r}\right]^{m}\right), & x>r\end{cases}
$$

as $m$ goes to the infinity. Moreover, $w_{m}$ is sufficiently smooth over the interval $(a, b)$; that is, $w_{m}(x) \in$ $C^{\infty}(a, b)$.

The generalized sigmoidal transformation $w_{m}$ plays an important role in developing a new approximation method as a weight function in this work.

\section{Decomposition of a Discontinuous Function}

From now on we suppose that $f$ is a piecewise smooth function containing a jump-discontinuity $\xi$ in an interval $[a, b]$. We assume that the location of $\xi$ or its accurate approximation is known and that the value $f(\xi)$ is defined to be the average of the left- and right-hand limits of $f$ at $\xi$; that is,

$$
f(\xi)=\frac{\{f(\xi-)+f(\xi+)\}}{2} .
$$

On the other side, taking $r=\xi$ in formula (1) or $w_{m}=$ $w_{m}(\xi ; x)$, we will use it as a weight function for the proposed approximation method in this work.

We choose the test functions below whose graphs are given in Figure 1:

$$
f_{1}(x)= \begin{cases}\frac{1}{5}(x-1)^{2}-4, & -\pi \leq x<\frac{\pi}{3} \\ x+\sin (3 x), & \frac{\pi}{3}<x \leq \pi\end{cases}
$$

which have a jump-discontinuity $\xi=\pi / 3$ :

$$
f_{2}(x)=\frac{1}{2}(x+1)^{2}, \quad-\pi \leq x \leq \pi
$$

which is continuous on the interval $[-\pi, \pi]$. We notice that $f_{i}(-\pi) \neq f_{i}(\pi), i=1,2$, and thus both $f_{1}$ and $f_{2}$ have jumpdiscontinuities at $\pm \pi$ when we extend these functions to the $2 \pi$ periodic functions over the real line.

Let the piecewise smooth function $f(x)$, containing a jump-discontinuity $\xi$, be defined as

$$
f(x)= \begin{cases}f_{L}(x), & a \leq x<\xi \\ f_{R}(x), & \xi<x \leq b,\end{cases}
$$

where $f_{L}$ and $f_{R}$ are continuous on $[a, \xi)$ and $(\xi, b]$, respectively. We assume that the order $m$ of $w_{m}=w_{m}(\xi ; x)$ is large 
enough throughout this paper. First, we define two quadratic polynomials $Q_{L}$ and $Q_{R}$ as

$$
Q_{L}(x)=\frac{a-\xi}{(b-\xi)^{2}}(x-\xi)^{2}+\xi
$$

satisfying

$$
\begin{aligned}
Q_{L}(\xi) & =\xi, \\
Q_{L}(b) & =a, \\
Q_{R}(x) & =\frac{b-\xi}{(a-\xi)^{2}}(x-\xi)^{2}+\xi
\end{aligned}
$$

satisfying

$$
\begin{aligned}
& Q_{R}(\xi)=\xi, \\
& Q_{R}(a)=b .
\end{aligned}
$$

It should be noted that the left- and right-hand limits of $Q_{L}$ and $Q_{R}$ at $\xi$ are

$$
\begin{aligned}
& Q_{L}(\xi-)=Q_{L}(\xi+)=\xi-, \\
& Q_{R}(\xi-)=Q_{R}(\xi+)=\xi+.
\end{aligned}
$$

Then we construct extensions of $f_{L}$ and $f_{R}$ onto the whole interval $[a, b]$ as

$$
\begin{aligned}
& \tilde{f}_{L}(x)=\left\{1-w_{m}(x)\right\} f(x)+w_{m}(x) f\left(Q_{L}(x)\right), \\
& \tilde{f}_{R}(x)=w_{m}(x) f(x)+\left\{1-w_{m}(x)\right\} f\left(Q_{R}(x)\right),
\end{aligned}
$$

respectively, for $a \leq x \leq b$. It is seen that

$$
\tilde{f}_{L}(\xi)=\tilde{f}_{R}(\xi)=f(\xi)=\frac{(f(\xi-)+f(\xi+))}{2}
$$

as $w_{m}(\xi)=1 / 2$.

One can surmise that, for sufficiently large $m, \tilde{f}_{L}$ has the effect of reflecting the left part $f_{L}$ of $f$ on $[a, \xi]$ into the opposite side $[\xi, b]$. So does $\tilde{f}_{R}$, symmetrically. In addition, these extended functions $\widetilde{f}_{L}$ and $\widetilde{f}_{R}$ defined in (14) and (15) have some particular properties as shown in the following lemmas.

Lemma 1. Let $f$ be a piecewise smooth function on $[a, b]$ with a jump-discontinuity $\xi$, and suppose that the order $m$ of $w_{m}$ is fixed and finite. Then we have the one-sided limits of $\tilde{f}_{L}$ and $\widetilde{f}_{R}$ as follows:

$$
\begin{aligned}
& \tilde{f}_{L}(\xi-)=f(\xi-), \\
& \tilde{f}_{L}\left(\xi_{+}\right)=\frac{\left(f(\xi-)+f\left(\xi_{+}\right)\right)}{2}, \\
& \tilde{f}_{R}\left(\xi_{-}\right)=\frac{\left(f(\xi-)+f\left(\xi_{+}\right)\right)}{2}, \\
& \tilde{f}_{R}\left(\xi_{+}\right)=f\left(\xi_{+}\right) .
\end{aligned}
$$

Furthermore,

$$
\begin{array}{ll}
\tilde{f}_{L}(a+)=\tilde{f}_{L}(b-) & (=f(a)), \\
\tilde{f}_{R}(a+)=\tilde{f}_{R}(b-) & (=f(b)) .
\end{array}
$$

Proof. Since $\lim _{x \rightarrow \xi} w_{m}(x)=1 / 2$ and $f\left(Q_{L}(\xi-)\right)=$ $f\left(Q_{L}\left(\xi_{+}\right)\right)=f\left(\xi_{-}\right)$for some $m$ fixed, from (14) we have

$$
\begin{aligned}
& \tilde{f}_{L}(\xi-)=\frac{1}{2}\left\{f(\xi-)+f\left(Q_{L}(\xi-)\right)\right\}=f(\xi-), \\
& \tilde{f}_{L}\left(\xi_{+}\right)=\frac{1}{2}\left\{f\left(\xi_{+}\right)+f\left(\xi_{-}\right)\right\} .
\end{aligned}
$$

By the same way, from (15) we have $\tilde{f}_{R}(\xi-)=(1 / 2)\{f(\xi-)+$ $\left.f\left(\xi_{+}\right)\right\}$and $\tilde{f}_{R}\left(\xi_{+}\right)=f\left(\xi_{+}\right)$. The equations in (18) directly result from the properties of $Q_{L}, Q_{R}$, and $w_{m}$.

Properties (17) in Lemma 1 imply that both $\tilde{f}_{L}$ and $\tilde{f}_{R}$ have the jump-discontinuity at $\xi$ if the original function $f$ has a jump-discontinuity such as $f(\xi-) \neq f(\xi+)$. The properties in (18) may resolve the troublesome problem in Fourier series approximation resulting from the mismatch at the end points.

In Figure 2, graphs of $\tilde{f}_{L}$ and $\widetilde{f}_{R}$ for the test function $f(x)=f_{1}(x)$ with $m=40$, for example, illustrate the results in Lemma 1. Therein, thick lines indicate principal part $f_{L}(x)$ of the extended functions $\widetilde{f}_{L}$ in (a) and $f_{R}(x)$ of $\widetilde{f}_{R}$ in (b). Thin lines indicate reflected parts of $f_{L}(x)$ and $f_{R}(x)$ in (a) and (b), respectively, and dotted lines show the original graph of $f(x)$.

For sufficiently large $m$, however, we can see that the jump-discontinuities of $\widetilde{f}_{L}$ and $\widetilde{f}_{R}$ at $\xi$ vanish as shown in the following lemma.

Lemma 2. For a function $f$ assumed in Lemma 1 both

$$
\begin{gathered}
\tilde{f}_{L}(\xi+)-\tilde{f}_{L}(\xi-), \\
\tilde{f}_{R}(\xi+)-\tilde{f}_{R}(\xi-)
\end{gathered}
$$

vanish as $m$ goes to the infinity.

Proof. It follows that $w_{m}(\xi-)=0$ and $w_{m}(\xi+)=1$ as $m \rightarrow \infty$. Thus from (14) and (15) we have $\tilde{f}_{L}\left(\xi_{-}\right)=\widetilde{f}_{L}\left(\xi_{+}\right)=f\left(\xi_{-}\right)$ and $\tilde{f}_{R}(\xi-)=\widetilde{f}_{R}\left(\xi_{+}\right)=f\left(\xi_{+}\right)$. The proof is completed.

Lemma 2 indicates the asymptotic behavior of $\tilde{f}_{L}$ and $\tilde{f}_{R}$ below:

$$
\begin{aligned}
& \tilde{f}_{L}(x) \sim \tilde{f}_{L, \infty}(x):= \begin{cases}f_{L}(x), & a \leq x \leq \xi \\
f_{L}\left(Q_{L}(x)\right), & \xi<x \leq b,\end{cases} \\
& \tilde{f}_{R}(x) \sim \tilde{f}_{R, \infty}(x):= \begin{cases}f_{R}\left(Q_{R}(x)\right), & a \leq x<\xi \\
f_{R}(x), & \xi \leq x \leq b\end{cases}
\end{aligned}
$$

for $m$ large enough. It should be noted that

$$
\begin{aligned}
& \lim _{x \rightarrow \xi} \tilde{f}_{L, \infty}(x)=f(\xi-), \\
& \lim _{x \rightarrow \xi} \tilde{f}_{R, \infty}(x)=f(\xi+) .
\end{aligned}
$$

Thus, if we replace the values of $f_{L}$ and $f_{R}$ at $\xi$ as $f_{L}(\xi)=$ $f(\xi-)$ and $f_{R}(\xi)=f(\xi+)$, then both $\widetilde{f}_{L, \infty}$ and $\widetilde{f}_{R, \infty}$ are continuous on the whole interval $[a, b]$. 


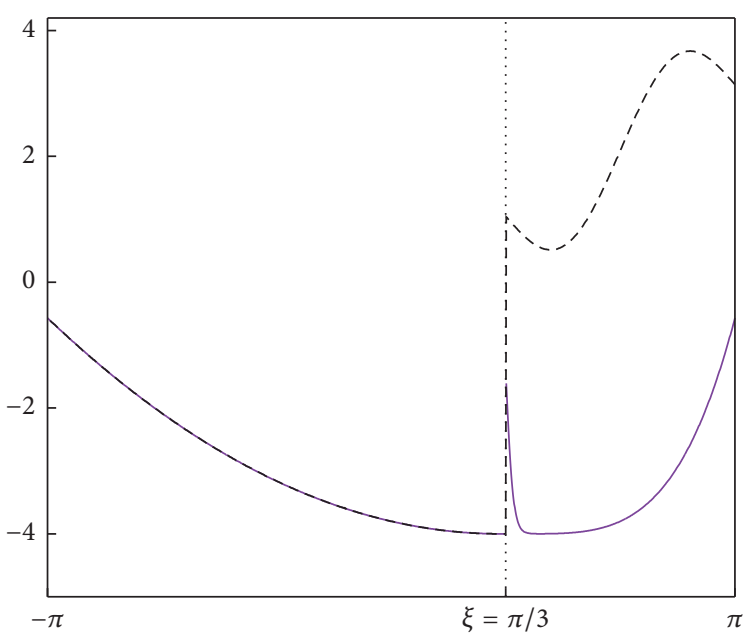

$(x)$

(a) $\tilde{f}_{L}(x)$

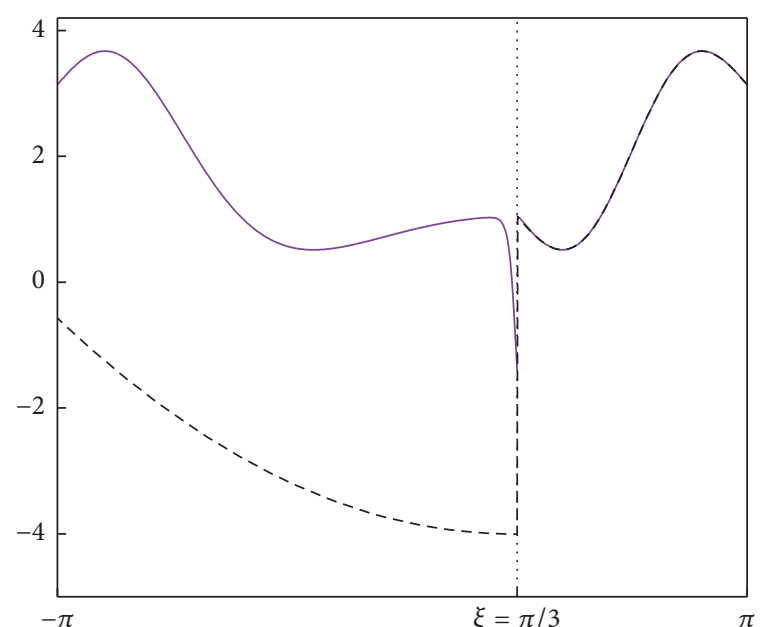

$(x)$

(b) $\tilde{f}_{R}(x)$

FIGURE 2: Graphs of the extended functions $\tilde{f}_{L}(x)$ and $\tilde{f}_{R}(x)$ with $m=40$ for the test function $f_{1}(x)$.

\section{Improving Fourier Partial Sum Approximation}

In this section we assume that the piecewise smooth function $f$ is defined on $[a, b]=[-\pi, \pi]$ with a jump-discontinuity $-\pi<\xi<\pi$. We consider Fourier series of $\widetilde{f}_{L}$ and $\tilde{f}_{R}$ in the form of

$$
\begin{aligned}
& S_{n} \tilde{f}_{L}(x)=\sum_{k=-n}^{n} c_{k}^{L} e^{i k x}, \\
& S_{n} \tilde{f}_{R}(x)=\sum_{k=-n}^{n} c_{k}^{R} e^{i k x},
\end{aligned}
$$

where $c_{k}^{L}$ and $c_{k}^{R}$ are Fourier coefficients defined as

$$
\begin{aligned}
& c_{k}^{L}=\int_{-\pi}^{\pi} \tilde{f}_{L}(t) e^{-i k t} d t, \\
& c_{k}^{R}=\int_{-\pi}^{\pi} \tilde{f}_{R}(t) e^{-i k t} d t .
\end{aligned}
$$

Then we propose a weighted average of $S_{n} \widetilde{f}_{L}$ and $S_{n} \widetilde{f}_{R}$ as follows:

$$
\begin{aligned}
F_{n}(x) & =\left\{1-w_{m}(x)\right\} S_{n} \tilde{f}_{L}(x)+w_{m}(x) S_{n} \tilde{f}_{R}(x) \\
& =\sum_{k=-n}^{n}\left\{c_{k}^{L}+\left(c_{k}^{R}-c_{k}^{L}\right) w_{m}(x)\right\} e^{i k x}
\end{aligned}
$$

for $-\pi \leq x \leq \pi$. It is noted that, like $\tilde{f}_{L}$ and $\tilde{f}_{R}$, the weighted average $F_{n}$ is discontinuous at $\xi$ if $f(\xi-) \neq f(\xi+)$. Nevertheless, $F_{n}$ has the meaningful convergence properties shown in the following theorem.

Theorem 3. Let $f$ be a function assumed in Lemma 1 with $[a, b]=[-\pi, \pi]$. Then we have the following:
(1) For the order $m$ of $w_{m}$ fixed, the weighted average $F_{n}(x)$ converges to

$$
\begin{gathered}
f(x)+O\left(h(x)^{m}\right), \\
h(x)=\min \left\{\frac{x+\pi}{\xi+\pi}, \frac{\pi-x}{\pi-\xi}\right\}
\end{gathered}
$$

pointwise over the interval $[-\pi, \pi]$, provided that the value of $F_{n}$ at the jump-discontinuity $\xi$ is defined as $F_{n}(\xi):=\left(F_{n}(\xi-)+F_{n}(\xi+)\right) / 2$.

(2) Let $F_{n, \infty}$ be a modified formula of $F_{n}$, in (26), obtained by replacing $\tilde{f}_{L}$ and $\widetilde{f}_{R}$ by their asymptotic versions $\widetilde{f}_{L, \infty}$ and $\widetilde{f}_{R, \infty}$ defined in (21) and (22), respectively, with the assumptions $f_{L}(\xi)=f(\xi-)$ and $f_{R}(\xi)=$ $f(\xi+)$. Then $F_{n, \infty}$ converges to $f$ pointwise over the interval $[-\pi, \pi]$, getting out of the Gibbs phenomenon, as $m, n \rightarrow \infty$.

Proof. It is noted that $S_{n} \widetilde{f}_{L}$ and $S_{n} \widetilde{f}_{R}$, respectively, converge to $\tilde{f}_{L}$ and $\tilde{f}_{R}$ pointwise on the interval $[-\pi, \pi]$ because $\tilde{f}_{L}$ and $\tilde{f}_{R}$ are both piecewise smooth [1].

Let $x<\xi$. Then from (26) and (5) we have

$\lim _{n \rightarrow \infty} F_{n}(x)$

$$
\begin{aligned}
& =\lim _{n \rightarrow \infty}\left\{S_{n} \tilde{f}_{L}(x)+\left[S_{n} \tilde{f}_{R}(x)-S_{n} \tilde{f}_{L}(x)\right] w_{m}(x)\right\} \\
& =f(x)+O\left(\left[\frac{x+\pi}{\xi+\pi}\right]^{m}\right) .
\end{aligned}
$$

Similarly, for $x>\xi$,

$$
\begin{aligned}
& \lim _{n \rightarrow \infty} F_{n}(x)=\lim _{n \rightarrow \infty}\left\{S_{n} \tilde{f}_{R}(x)\right. \\
& \left.+\left[S_{n} \widetilde{f}_{L}(x)-S_{n} \widetilde{f}_{R}(x)\right]\left(1-w_{m}(x)\right)\right\}=f(x) \\
& \quad+O\left(\left[\frac{\pi-x}{\pi-\xi}\right]^{m}\right) .
\end{aligned}
$$




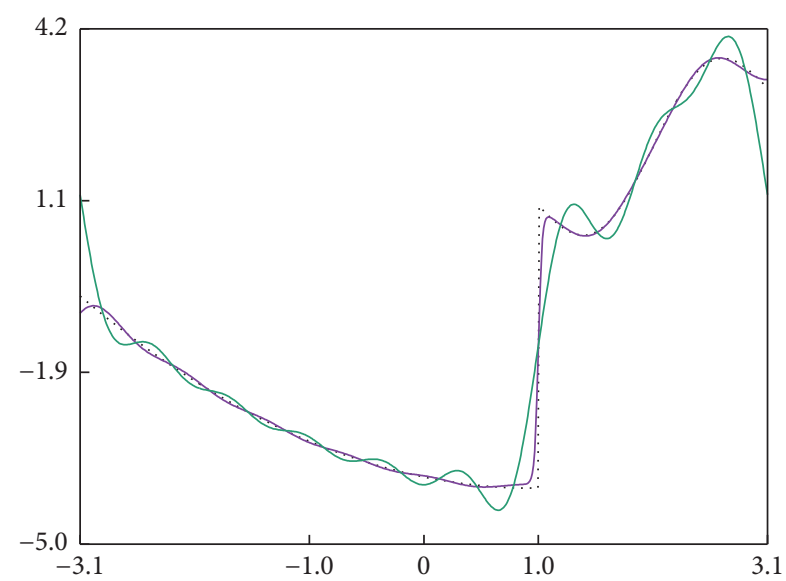

$(x)$

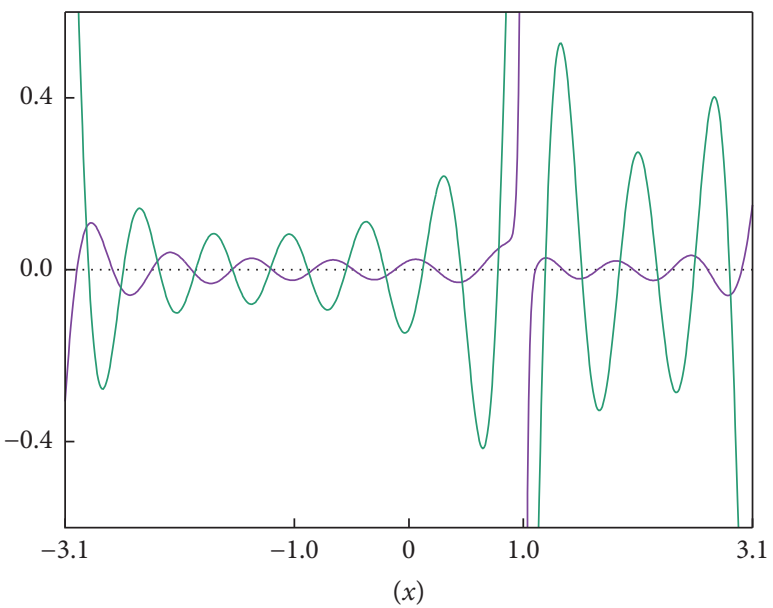

(a) $n=8$

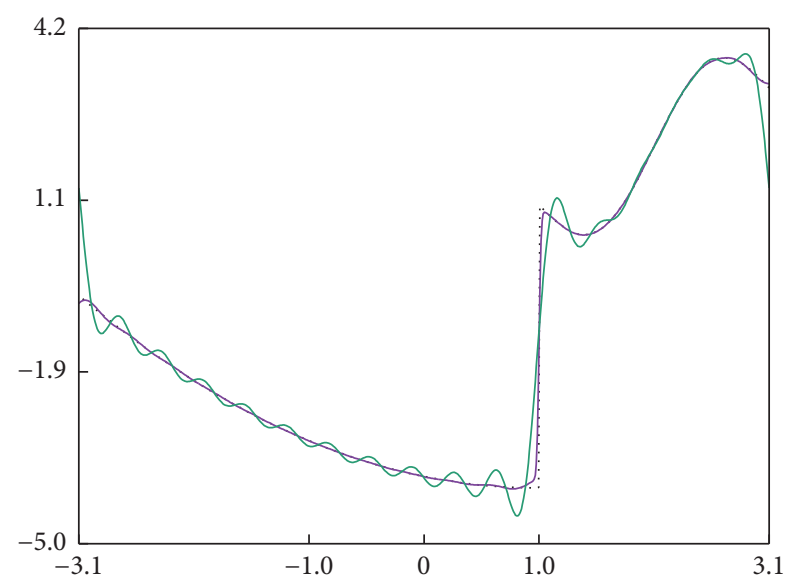

$(x)$

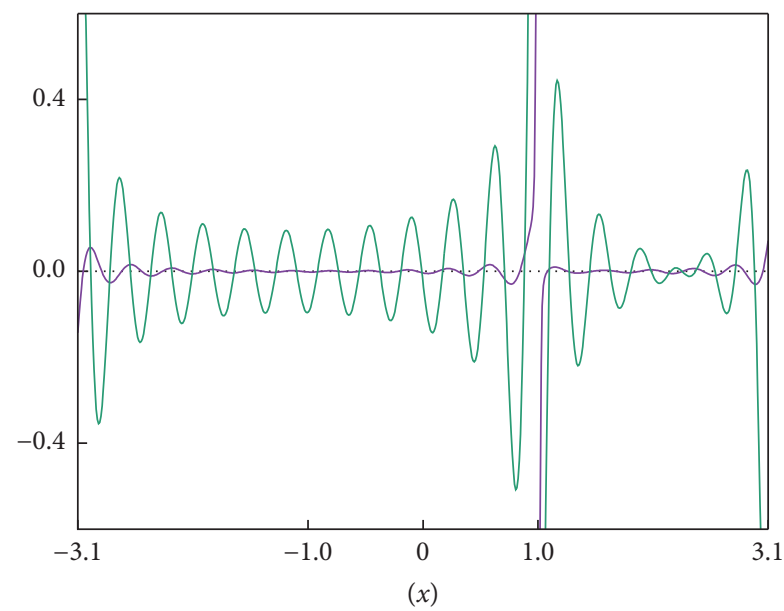

(b) $n=16$

FIGURE 3: Approximations of the weighted average $F_{n}(x)$ in upper rows and the corresponding errors in lower rows for the test function $f_{1}(x)$. Thin lines indicate approximations and errors of the Fourier partial sum $S_{n} f(x)$.

For $x=\xi$, from definition (26) of $F_{n}$ and results (17) in Lemma 1 , we have the equations

$$
\begin{aligned}
\lim _{n \rightarrow \infty} F_{n}(\xi-) & =\frac{1}{2}\left\{\tilde{f}_{L}(\xi-)+\tilde{f}_{R}(\xi-)\right\} \\
& =\frac{(3 f(\xi-)+f(\xi+))}{4}, \\
\lim _{n \rightarrow \infty} F_{n}(\xi+) & =\frac{1}{2}\left\{\tilde{f}_{L}(\xi+)+\tilde{f}_{R}(\xi+)\right\} \\
& =\frac{\left(f(\xi-)+3 f\left(\xi_{+}\right)\right)}{4} .
\end{aligned}
$$

This implies that

$$
\begin{aligned}
\lim _{n \rightarrow \infty} F_{n}(\xi) & =\lim _{n \rightarrow \infty} \frac{\left(F_{n}(\xi-)+F_{n}(\xi+)\right)}{2} \\
& =\frac{f(\xi-)+f(\xi+)}{2}=f(\xi) .
\end{aligned}
$$

Therefore, the proof of the assertion that $F_{n}$ converges to $f$ pointwise over the interval $[-\pi, \pi]$ is completed.

For (2), it is clear from assertion (1) that $F_{n, \infty}$ converges to $f$ pointwise over the interval $[-\pi, \pi]$ as $m, n \rightarrow \infty$. On the other hand, the definitions of $\tilde{f}_{L, \infty}$ and $\widetilde{f}_{R, \infty}$ in $(21)$ and (22), respectively, and the assumptions $f_{L}(\xi)=f(\xi-)$ and $f_{R}(\xi)=f(\xi+)$ imply that $\tilde{f}_{L, \infty}$ and $\tilde{f}_{R, \infty}$ are continuous at the original discontinuity $\xi$ with $\tilde{f}_{L, \infty}(-\pi)=\tilde{f}_{L, \infty}(\pi)$ and $\tilde{f}_{R, \infty}(-\pi)=\tilde{f}_{R, \infty}(\pi)$. That is, $\tilde{f}_{L, \infty}$ and $\tilde{f}_{R, \infty}$ are free of jumpdiscontinuity at $x=\xi, \pm \pi$. Thus, the Fourier series $S_{n} \tilde{f}_{L, \infty}$ and $S_{n} \widetilde{f}_{R, \infty}$ uniformly converge to $\widetilde{f}_{L, \infty}$ and $\widetilde{f}_{R, \infty}$, respectively. As a result, we can see that the weighted combination $F_{n, \infty}$ of $S_{n} \widetilde{f}_{L, \infty}$ and $S_{n} \widetilde{f}_{R, \infty}$ will get out of the Gibbs phenomenon as $m, n \rightarrow \infty$. This completes the proof.

Results of the approximations and errors of $F_{n}(x)$ with $n=8,16$, for the test functions $f_{1}$ and $f_{2}$, are illustrated in Figures 3 and 4, respectively. Therein, we took the order of weight function as $m=10 n$, for example. The results 


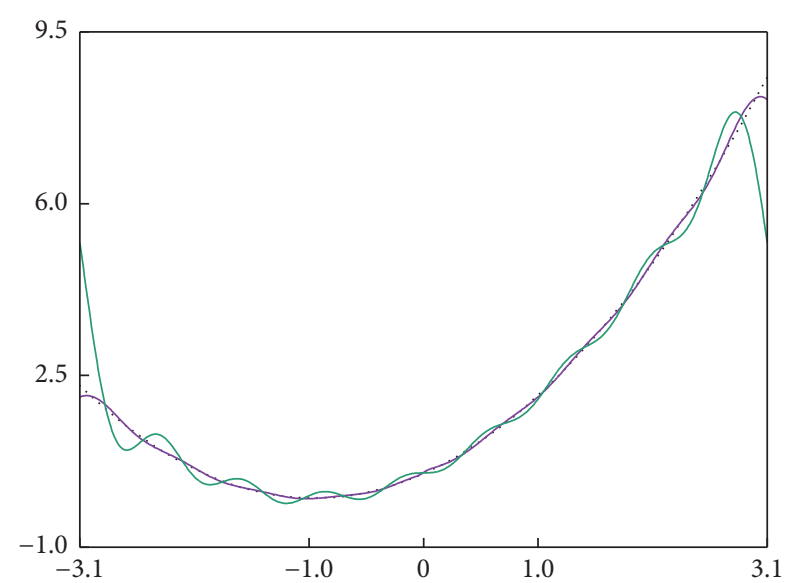

$(x)$

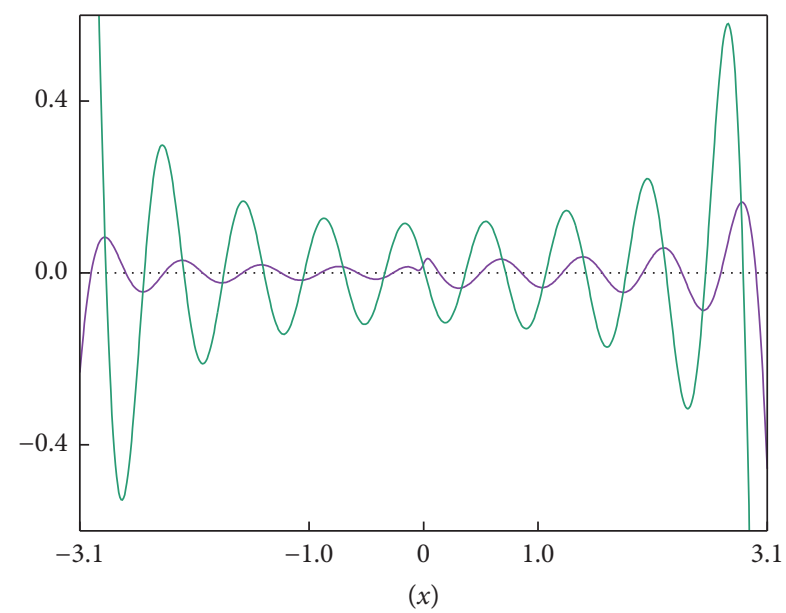

(a) $n=8$

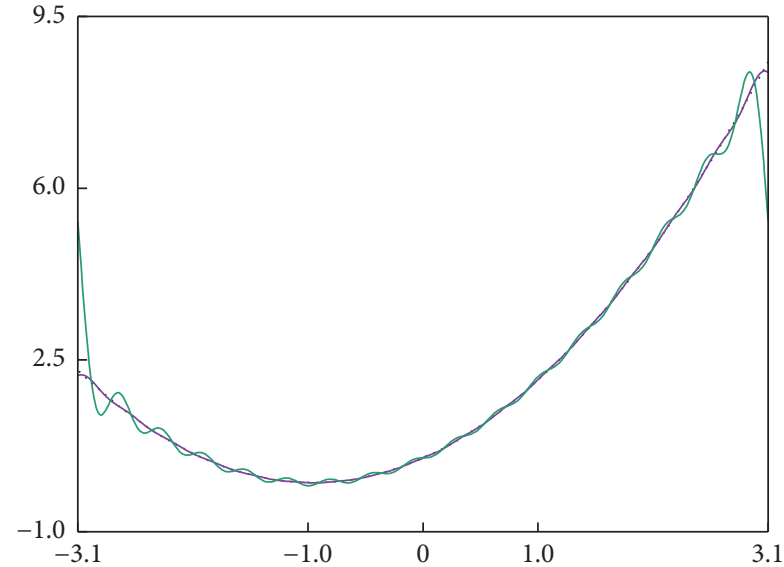

$(x)$

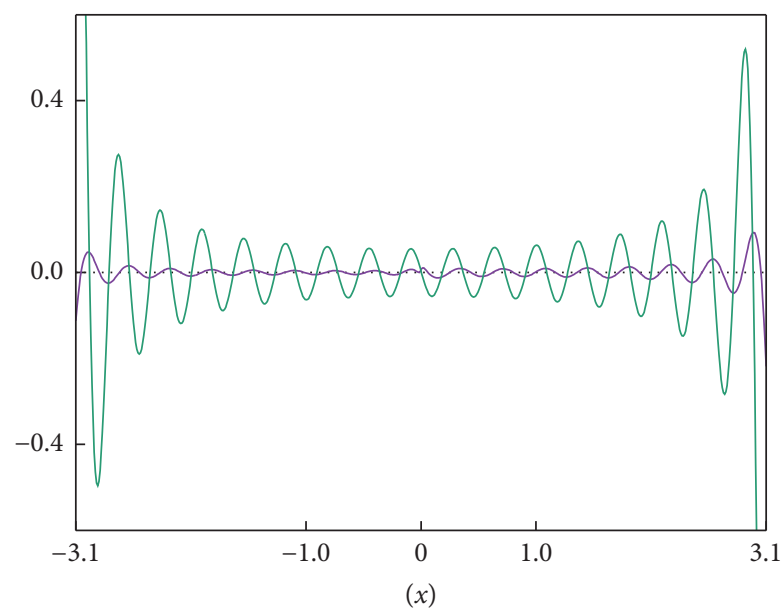

(b) $n=16$

FIGURE 4: Approximations of the weighted average $F_{n}(x)$ in upper rows and the corresponding errors in lower rows for the test function $f_{2}(x)$. Thin lines indicate approximations and errors of the Fourier partial sum $S_{n} f(x)$.

of $F_{n}(x)$, indicated by the thick lines, are compared with those of the traditional Fourier partial sum approximation $S_{n} f(x)$ which are indicated by the thin lines. The figures show that the proposed approximation $F_{n}(x)$ highly improves the Fourier partial sum approximation over the whole interval. In particular, the Gibbs phenomenon resulting from the interior jump-discontinuity or the mismatch at the end points has been resolved by $F_{n}(x)$.

\section{Conflicts of Interest}

The author declares that he has no conflicts of interest regarding the publication of this paper.

\section{Acknowledgments}

This research was supported by Basic Science Research Program through the National Research Foundation of Korea (NRF) funded by the Ministry of Science and ICT (NRF2017R1A2B4007682).

\section{References}

[1] G. B. Folland, Fourier analysis and Its applications, Wadsworth \& Brooks Cole Advanced Books \& Software, Pacific Grove, Calif, USA, 1992.

[2] A. J. Jerry, The Gibbs phenomenon in Fourier Analysis, Splines and Wavelet Approximations, Kluwer Academic Publ, London, UK, 1998.

[3] J. P. Boyd, "Trouble with GEGenbauer reconstruction for defeating GIBbs' phenomenon: Runge phenomenon in the diagonal limit of GEGenbauer polynomial approximations," Journal of Computational Physics, vol. 204, no. 1, pp. 253-264, 2005.

[4] A. Gelb and D. Gottlieb, "The resolution of the Gibbs phenomenon for spliced functions in one and two dimensions," Computers \& Mathematics with Applications, vol. 33, no. 11, pp. 35-58, 1997.

[5] D. Gottlieb and C.-W. Shu, "On the Gibbs phenomenon and its resolution," SIAM Review, vol. 39, no. 4, pp. 644-668, 1997.

[6] J.-H. Jung and B. D. Shizgal, "Generalization of the inverse polynomial reconstruction method in the resolution of the 
Gibbs phenomenon," Journal of Computational and Applied Mathematics, vol. 172, no. 1, pp. 131-151, 2004.

[7] B. D. Shizgal and J.-H. Jung, "Towards the resolution of the Gibbs phenomena," Journal of Computational and Applied Mathematics, vol. 161, no. 1, pp. 41-65, 2003.

[8] A. J. Jerri, "Lanczos-like $\sigma$-factors for reducing the Gibbs phenomenon in general orthogonal expansions and other representations," Journal of Computational Analysis and Applications, vol. 2, no. 2, pp. 111-127, 2000.

[9] E. Tadmor and J. Tanner, "Adaptive mollifiers for high resolution recovery of piecewise smooth data from its spectral information," Foundations of Computational Mathematics, vol. 2, no. 2, pp. 155-189, 2002.

[10] E. Tadmor and J. Tanner, "Adaptive filters for piecewise smooth spectral data," IMA Journal of Numerical Analysis (IMAJNA), vol. 25, no. 4, pp. 635-647, 2005.

[11] B. I. Yun, "A weighted averaging method for treating discontinuous spectral data," Applied Mathematics Letters, vol. 25, no. 9, pp. 1234-1239, 2012.

[12] B. I. Yun, "A cumulative averaging method for piecewise polynomial approximation to discrete data," Applied Mathematical Sciences, vol. 10, no. 5-8, pp. 331-343, 2016.

[13] S. Prössdorf and A. Rathsfeld, "On an integral equation of the first kind arising from a cruciform crack problem," in Integral Equations and Inverse Problems, V. Petkov and R. Lazarov, Eds., 1991.

[14] D. Elliott, "Sigmoidal transformations and the trapezoidal rule," The Journal of the Australian Mathematical Society B: Applied Mathematics, vol. 40, pp. E77-E137, 1998. 


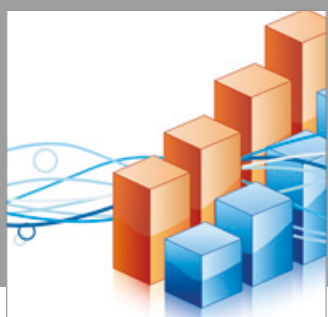

Advances in

Operations Research

vatersals

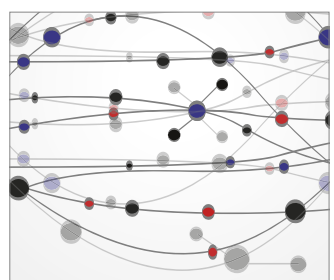

\section{The Scientific} World Journal
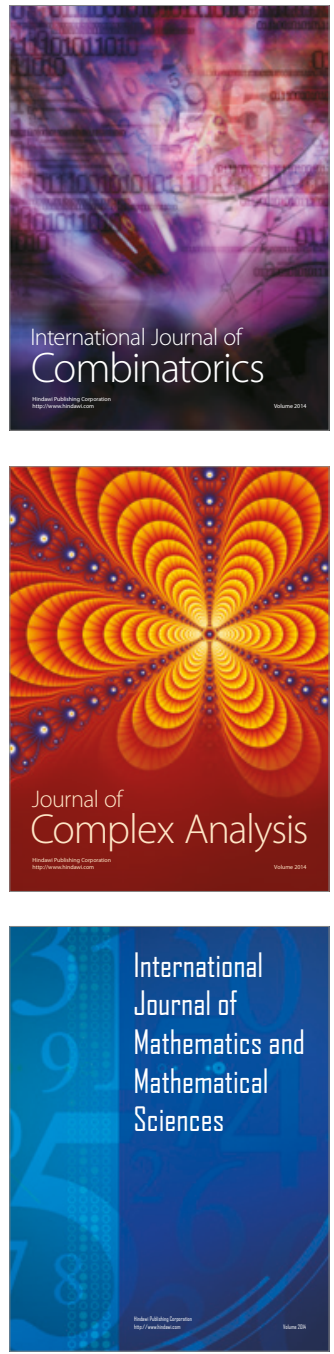
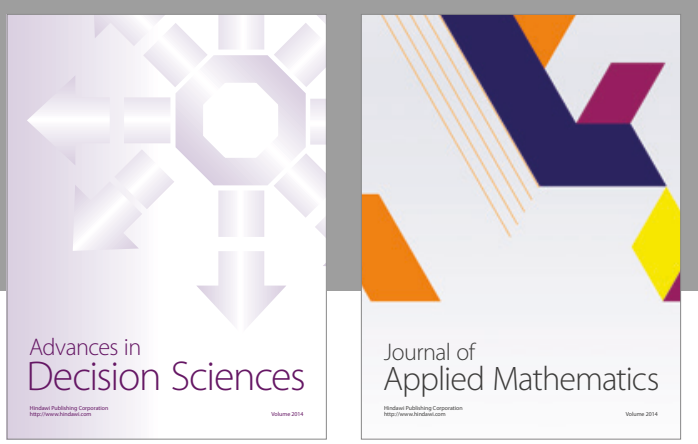

Algebra

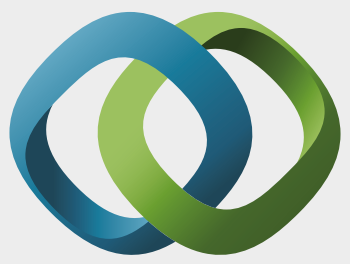

\section{Hindawi}

Submit your manuscripts at

https://www.hindawi.com
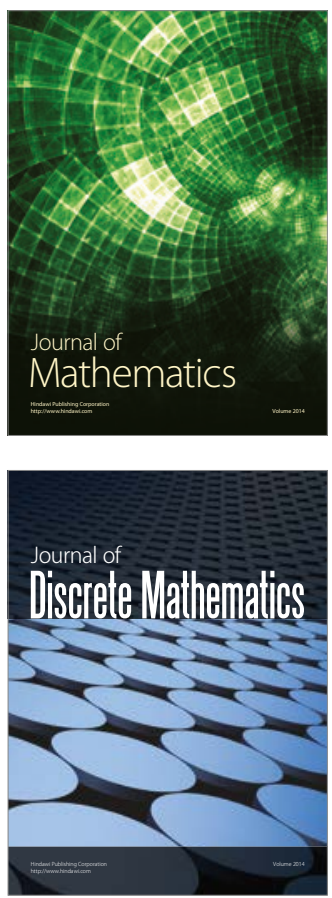

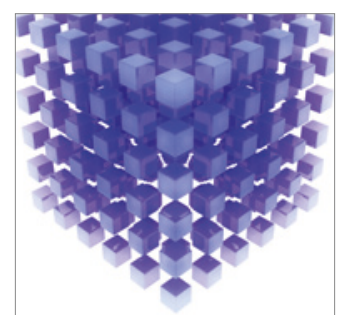

Mathematical Problems in Engineering
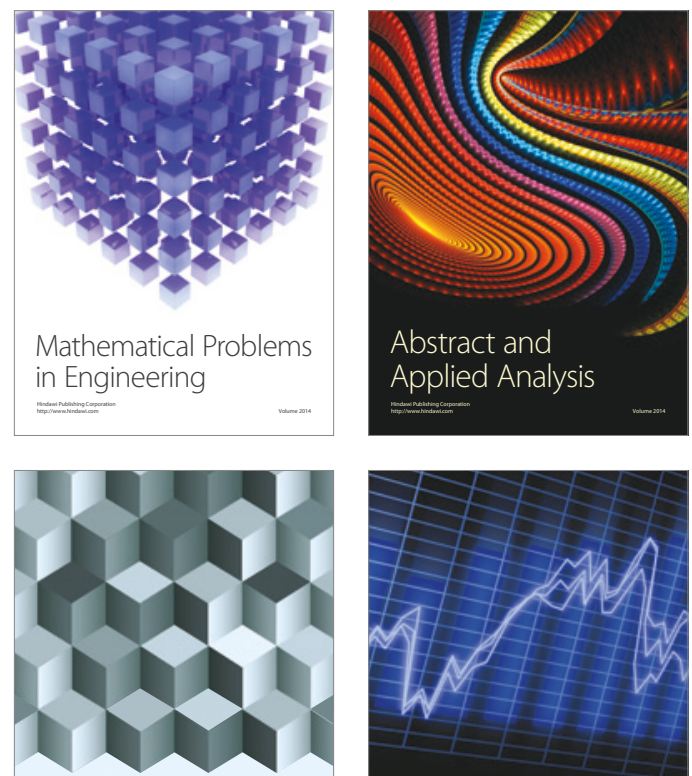

Journal of

Function Spaces

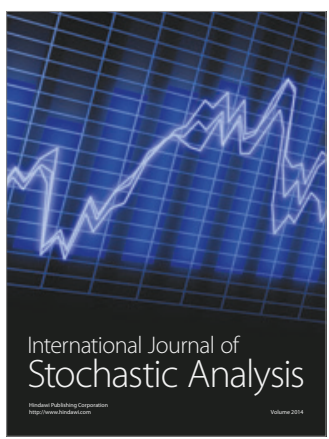

Probability and Statistics
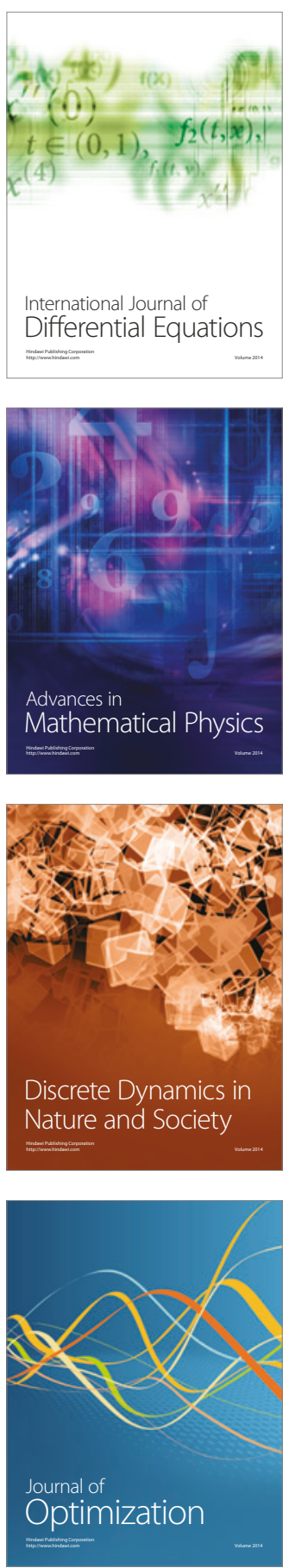